\title{
Computation-free presentation of the fundamental group of generic $(p, q)$-torus curves
}

\author{
ENRIQUE ARTAL BARTOLO \\ José Ignacio Cogolludo Agustín \\ JORGE ORTIGAS-GALINDO
}

\begin{abstract}
We present a new method for computing fundamental groups of curve complements using a variation of the Zariski-van Kampen method on general ruled surfaces. As an application we give an alternative (computation-free) proof for the fundamental group of generic $(p, q)$-torus curves.
\end{abstract}

14F45, 14H30, 14H50; 57M05, 57M12, 14H10, 14E05

\section{Introduction}

O Zariski [9] computed the fundamental group of the complement in $\mathbb{P}^{2}$ of the discriminant curve of the projection of a generic cubic surface in $\mathbb{P}^{3}$ onto $\mathbb{P}^{2}$. Such group turns out to be $\mathbb{Z} / 2 \mathbb{Z} * \mathbb{Z} / 3 \mathbb{Z}$ and this is related to the fact that the equation of such a curve is of the type $f_{2}^{3}+f_{3}^{2}=0$, where $f_{m}$ is a homogeneous polynomial of degree $m$ in three variables. Even if not all the details are fully justified in that paper, the result is true and the techniques therein are behind the well-known Zariski-van Kampen method $[9 ; 8]$.

Following these ideas, M Oka [7] proved that the fundamental group of the complement of the projective curve with equation $\left(x^{p}+y^{p}\right)^{q}+\left(y^{q}+z^{q}\right)^{p}=0$ is isomorphic to $\mathbb{Z} / p \mathbb{Z} * \mathbb{Z} / q \mathbb{Z}$, for $p, q$ coprime. In order to obtain this result the author is forced to perform long computations as a result of using the Zariski-van Kampen method (see [6] for another approach given by A Némethi). By standard arguments, the same computations used by Oka are true for the complement of $f_{p}^{q}+f_{q}^{p}=0$, which will be called from now on a $(p, q)$-torus curve, for generic $f_{p}$ and $f_{q}$ : there is an isotopy from any generic $(p, q)$-torus curve to Oka's curve. Therefore the fundamental group of the complement of $f_{p}^{q}+f_{q}^{p}=0$ is isomorphic to $\mathbb{Z} / p \mathbb{Z} * \mathbb{Z} / q \mathbb{Z}$ as well.

In this note, we present a new method to compute fundamental groups of complements of curves via Nagata transformations and fibrations from ruled rational surfaces. This 
way we give an alternative computation for the fundamental group of generic $(p, q)-$ torus curves.

The idea is to use the first part to compute the fundamental group in a degenerated case and then use orbifold fundamental group techniques to recover the generic case.

Acknowledgements All authors are partially supported by the Spanish Ministry of Education grant MTM2010-21740-C02-02.

\section{Zariski-van Kampen Method on ruled surfaces}

We will describe this general method in a particular example using the idea of the classical Zariski-van Kampen Method on Hirzebruch surfaces.

Let $N:=2 m+1$ be an odd number coprime with $d:=a+b, a, b \in \mathbb{N}$ coprime. We consider the plane projective curve $\mathcal{C}:=\mathcal{C}_{N, a, b}$ defined by the following equation:

$$
F_{N, a, b}(x, y, z):=x^{a N} y^{b N}+\left(x^{N}+y^{N}+x^{m} y^{m} z\right)^{d}=0 .
$$

This curve has degree $d N$. Note that $P:=[0: 0: 1]$ is a singular point of $\mathcal{C}$. Let us consider the pencil of lines passing through $P$. We denote them by $L_{t}, t \in \mathbb{P}^{1} \equiv$ $\mathbb{C} \cup\{\infty\}$, where

$$
L_{t}:= \begin{cases}\{y-t x=0\} & \text { if } t \in \mathbb{C} \\ \{x=0\} & \text { if } t=\infty .\end{cases}
$$

The lines $L_{0}$ and $L_{\infty}$ intersect $\mathcal{C}$ only at $P$. Since the multiplicity of $(\mathcal{C}, P)$ equals $2 m d=(N-1) d$, a generic line $L_{t}$ intersect $\mathcal{C}$ at $N d-(N-1) d=d$ points outside $P$. The tangent cone of $(\mathcal{C}, P)$ consists of the two lines $L_{0}$ and $L_{\infty}$. In order to study this singularity we perform a blowing-up at $P$. With suitable charts the local equation of the strict transform of the branches tangent to $L_{0}$ are of the form

$$
x^{a N} y^{d}+\left(x^{N} y+y+x^{m}\right)^{d}=0,
$$

which is tangent to $y=0$ (as far as $m>1$ ), the equation of the exceptional divisor on this chart. Let $y_{1}:=y+x^{m}$. Then (1-2) becomes

$$
x^{a N}\left(y_{1}-x^{m}\right)^{d}+\left(y_{1}+x^{N} y_{1}-x^{N+m}\right)^{d}=0 .
$$

Looking at its Newton polygon, we deduce that such a singularity is topologically equivalent to $y_{1}^{d}+x^{a N+m d}=0$. Since $\operatorname{gcd}(d, a N+m d)=1$, it is irreducible.

Something analogous occurs when considering the branches at the infinitely near point associated with the tangent direction $L_{\infty}$. Blowing-down, we can describe the topological type of the original singularity $(\mathcal{C}, P)$. 
Lemma 1.1 The singularity $(\mathcal{C}, P)$ has two branches. The branch tangent to $L_{0}$ is of type $(a N+m d, a N+(m+1) d)$ and the branch tangent to $L_{\infty}$ is of type $(b N+(m+1) d, b N+m d)$.

Using Riemann-Hurwitz arguments, any other line through $P$ intersects $\mathcal{C}$ transversally outside $P$ and in particular $P$ is the only singular point of $\mathcal{C}$.

We want to compute the fundamental group of $\mathbb{P}^{2} \backslash \mathcal{C}$ using a generalized Zariskivan Kampen method where $P$ is the projection point. For the classical Zariski-van Kampen method the projection point is a generic point outside $\mathcal{C}$. In our case, not only $P \in \mathcal{C}$ but also the tangent cone of $(\mathcal{C}, P)$ consists of two lines; hence, for any choice of line at infinity we should deal with vertical asymptotes and this is a strong technical problem. In order to deal with these issues, we are going to perform Nagata's elementary operations to some ruled surfaces. Since it will be more useful for our purposes, we replace Nagata's elementary operations by a sequence of blowing-ups and blowing-downs.

Let $\sigma_{0}: \Sigma_{1} \rightarrow \mathbb{P}^{2}$ be the blowing-up of $\mathbb{P}^{2}$ at $P$ and denote by $E:=\sigma_{0}^{-1}(P)$ the exceptional divisor. The divisor $E$ is a $(-1)$-curve which is a section of the ruling $\Pi_{1}: \Sigma_{1} \rightarrow \mathbb{P}^{1}$.

Convention 1.2 For the sake of simplicity, given a blowing-up, the strict transform of a curve will keep the same notation.

One has the following properties:

$\left(\Sigma_{1} 1\right) \quad \sigma_{0}^{-1}(\mathcal{C})=\mathcal{C} \cup E$.

$\left(\Sigma_{1} 2\right) \quad L_{0}$ and $L_{\infty}$ are the only fibers of $\Pi_{1}$ which are nontransversal to $\mathcal{C}$ (and to $\mathcal{C} \cup E)$.

Figure 1 describes the standard ruling $\Pi_{1}$ of $\Sigma_{1}$. The number in brackets after a divisor represents its self-intersection. This is not yet a good model for any Zariskivan Kampen based method since the curve $\mathcal{C}$ intersects the negative curve $E$. Let $P_{t}:=L_{t} \cap E$. Following Lemma 1.1 and (1-2)-(1-3), we deduce that $\left(\mathcal{C}, P_{\infty}\right)$ is a singular point of type $(a N+m d, d)$, the curve $E$ is tangent to $\mathcal{C}$ and $(\mathcal{C} \cdot E)_{P_{\infty}}=m d$.

Analogously $\left(\mathcal{C}, P_{0}\right)$ is a singular point of type $(b N+m d, d)$, the curve $E$ is tangent to $\mathcal{C}$ and $(\mathcal{C} \cdot E)_{P_{0}}=m d$. To separate $E$ and $\mathcal{C}$ we perform $m$ blowing-ups at $P_{\infty}=P_{\infty}^{0}$ (and the following $(m-1)$ infinitely near points $P_{\infty}^{j}$ at $\left.E, j=1, \ldots, m-1\right)$, where $P_{\infty}^{j}:=\mathcal{C} \cap E_{\infty}^{j}$ and $E_{\infty}^{j}$ is the exceptional divisor obtained after blowing up $P_{\infty}^{j-1}$. Also, note that the multiplicity of $\mathcal{C}$ at $P_{\infty}^{j}$ is $d$. Note that the point $P_{\infty}^{m}$ is not on $E$ 


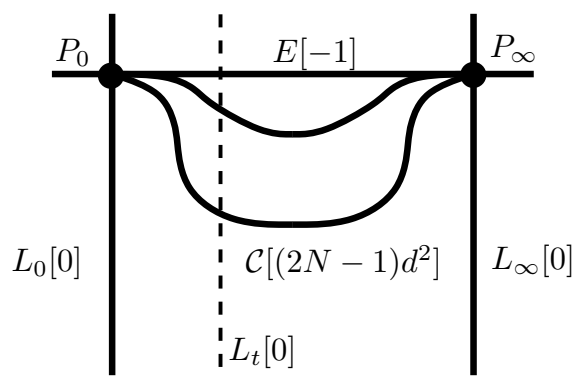

Figure 1. Projection after the blowing-up of $P$

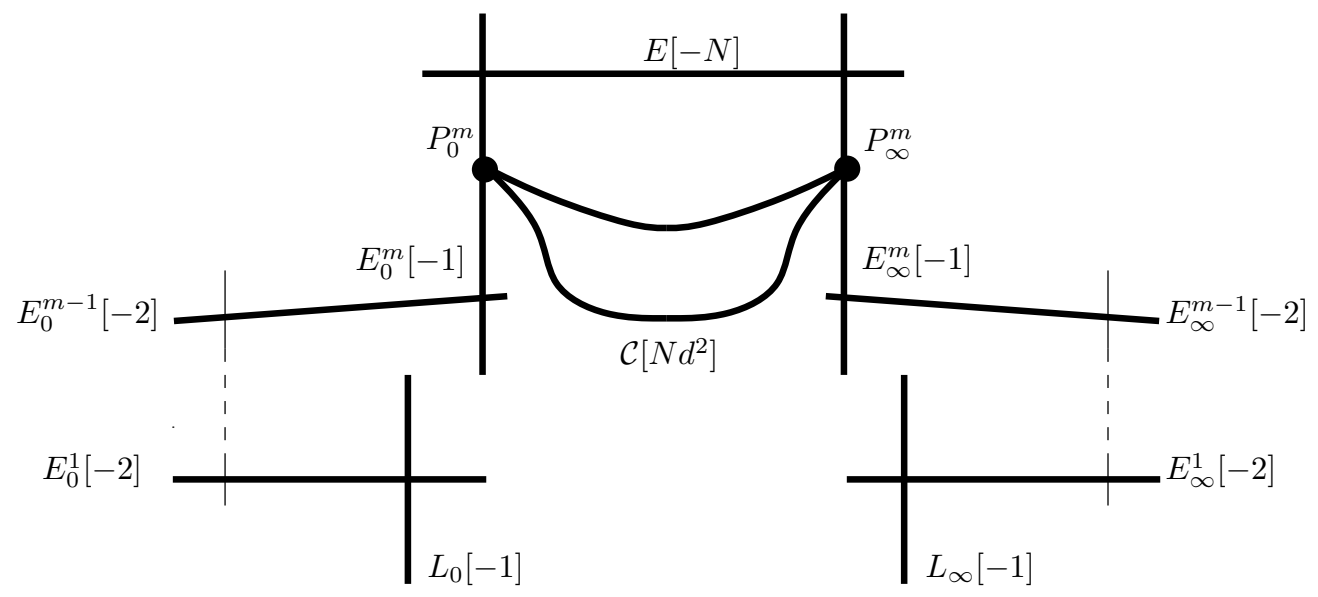

Figure 2. The surface $\widehat{\Sigma}$

anymore. One repeats this procedure for $P_{0}=P_{0}^{0}$. This way a surface $\hat{\Sigma}$ is obtained as shown in Figure 2. Now, we can blow down the curves $L_{\infty}, E_{\infty}^{1}, \ldots, E_{\infty}^{m-1}$ and $L_{0}, E_{0}^{1}, \ldots, E_{0}^{m-1}$ in order to obtain a ruled surface $\Sigma_{N}$ where $\mathcal{C}$ is disjoint to $E$. The singularity type of $\left(\mathcal{C}, P_{\infty}^{m}\right)$ is $(a N, d)$ and it is transversal to $E_{\infty}^{m}$. Analogously, the singularity type of $\left(\mathcal{C}, P_{0}^{m}\right)$ is $(b N, d)$ and it is transversal to $E_{0}^{m}$.

Proposition 1.3 The monodromy action on $\Sigma_{N}$ is given as follows. Let $L_{t}$ be a generic fiber. We can choose meridians $\mu_{0}, \mu_{\infty}$, and $\mu_{t}$ on $\mathbb{P}^{1} \backslash\left\{p_{0}, p_{\infty}, p_{t}\right\}$ around the images $p_{0}, p_{\infty}, p_{t}$ of the fibers $E_{0}^{m}, E_{\infty}^{m}, L_{t}$ of $\Pi_{N}: \Sigma_{N} \rightarrow \mathbb{P}^{1}$, such that $\mu_{0} \cdot \mu_{\infty} \cdot \mu_{t}=1 \in \pi_{1}\left(\mathbb{P}^{1} \backslash\left\{p_{0}, p_{\infty}, p_{t}\right\}\right)$. For these meridians, the braid monodromy is given by

$$
\mu_{0} \mapsto \beta_{0}=\left(\sigma_{d-1} \cdots \sigma_{1}\right)^{a N}, \quad \mu_{\infty} \mapsto \beta_{\infty}=\left(\sigma_{d-1} \cdots \sigma_{1}\right)^{b N},
$$

where the $\sigma_{1}, \ldots, \sigma_{d-1}$ are appropriate half twists generating the braid group $\mathbb{B}_{d}$ on $L_{t}$ based on the set $L_{t} \cap \mathcal{C}$. 
Proof The braid monodromy is defined over $\mathbb{P}^{1} \backslash\left\{p_{0}, p_{\infty}, p_{t}\right\}$ (which is like a twopunctured $\mathbb{C}$ ). We need to take out a fiber, say $L_{t}$, in order to have a trivialization that allows one to define the braids. From the topological type of the singularity at $P_{0}^{m}$ we know that the image $\beta_{0}$ of $\mu_{0}$ can be chosen to be a conjugate of $\left(\sigma_{d-1} \cdots \sigma_{1}\right)^{a N}$, hence after an appropriate choice of the generators $\sigma_{i}$, the image $\beta_{0}$ can be set to be exactly $\left(\sigma_{d-1} \cdots \sigma_{1}\right)^{a N}$. Once the generators $\sigma_{i}$ are fixed and using the topological type of the singularity $P_{\infty}^{m}$, the image $\beta_{\infty}$ of $\mu_{\infty}$ will be a conjugate of $\left(\sigma_{d-1} \cdots \sigma_{1}\right)^{b N}$. Since $L_{t}$ is generic, we are in $\Sigma_{N}$ and we are avoiding the negative section $E$, we have that the image of $\mu_{t}^{-1}=\mu_{\infty} \cdot \mu_{0}$ is $\Delta^{2 N}$, where $\Delta^{2}=\left(\sigma_{d-1} \cdots \sigma_{1}\right)^{d}$ is the positive generator of the center of $\mathbb{B}_{d}$; see Kharlamov and Kulikov [5, Lemma 2.1]. Hence, $\beta_{\infty}=\left(\sigma_{d-1} \cdots \sigma_{1}\right)^{b N}$ is as in the statement.

Note that

(1-5) $\mathbb{P}^{2} \backslash\left(\mathcal{C} \cup L_{0} \cup L_{\infty}\right) \cong \Sigma_{1} \backslash\left(\mathcal{C} \cup E \cup L_{0} \cup L_{\infty}\right) \cong \Sigma_{N} \backslash\left(\mathcal{C} \cup E \cup E_{0}^{m} \cup E_{\infty}^{m}\right)$.

Lemma 1.4 The meridians of $L_{\infty}$ and $E_{\infty}^{m}$ (resp. $L_{0}$ and $E_{0}^{m}$ ) are conjugate in $\widehat{\Sigma}$. As a consequence,

$$
\pi_{1}\left(\mathbb{P}^{2} \backslash \mathcal{C}\right) \cong \pi_{1}\left(\Sigma_{N} \backslash(\mathcal{C} \cup E)\right)
$$

Proof Let us consider the sequence of blowing-ups used to obtain Figure 2 from Figure 3. Note that if we blow up a smooth point on a divisor $D$, a meridian of

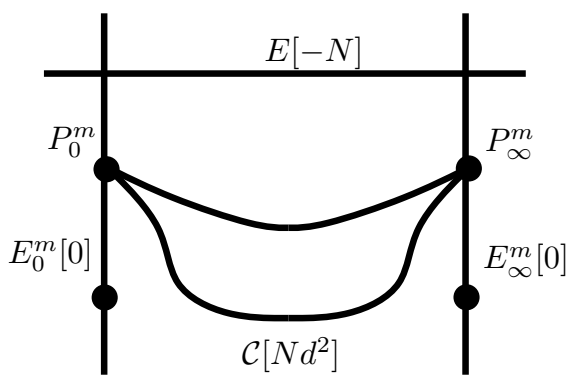

Figure 3. The situation on $\Sigma_{N}$

the exceptional component equals a meridian of $D$. This gives the first statement. The second part is a consequence of (1-5) and the following well-known fact: let $S$ be a quasiprojective surface and let $A$ be an irreducible curve in $S$, then the map $\pi_{1}(S \backslash A) \rightarrow \pi_{1}(S)$ is surjective and its kernel is normally generated by a meridian of $A$.

Theorem $1.5 \pi_{1}\left(\mathbb{P}^{2} \backslash \mathcal{C}\right) \cong \mathbb{Z} / d \mathbb{Z} * \mathbb{Z} / N \mathbb{Z}$. 
Proof The Zariski-van Kampen method on $\Sigma_{N} \backslash(\mathcal{C} \cup E)$ provides a way to obtain a presentation of $\pi_{1}\left(\Sigma_{N} \backslash(\mathcal{C} \cup E)\right)$ as follows. Let us define $\beta:=\left(\sigma_{d-1} \cdots \sigma_{1}\right)^{N}$. According to this, $\beta_{0}=\beta^{a}$ and $\beta_{\infty}=\beta^{b}$ in (1-4). Then,

$$
\begin{aligned}
\pi_{1}\left(\Sigma_{N}\right. & \backslash(\mathcal{C} \cup E)) \\
& =\left\langle\mu_{1}, \ldots, \mu_{d} \mid \mu_{i}=\mu_{i}^{\beta_{0}}, \mu_{i}=\mu_{i}^{\beta_{\infty}}, 1 \leq i \leq d,\left(\mu_{d} \cdots \mu_{1}\right)^{N}=1\right\rangle .
\end{aligned}
$$

Since $\operatorname{gcd}(a, b)=1$, the first two sets of relations in (1-6) can be replaced by $\mu_{i}=\mu_{i}^{\beta}$, $1 \leq i \leq d$. Since by hypothesis $\operatorname{gcd}(d, N)=1$, these are exactly the relations of the fundamental group of torus knot of type $(d, N)$, which is given by $A^{d}=B^{N}$ for $B=\mu_{d} \cdots \mu_{1}$. The last relation in (1-6) implies $B^{N}=1$, hence the result follows.

\section{Torus curves}

Definition 2.1 A $(p, q)$-torus curve, $\operatorname{gcd}(p, q)=1$, is a curve $\mathcal{C}$ admitting an equation $f_{p}^{q}+f_{q}^{p}=0$, where $f_{m}$ is a homogeneous polynomial of degree $m$ in three variables. We say that $\mathcal{C}$ is a generic torus curve if the curves of equation $f_{p}=0$ and $f_{q}=0$ intersect transversally at $p q$ distinct points and they are the only singular points of $\mathcal{C}$.

The following result is straightforward.

Proposition 2.2 Let $\mathcal{C}_{0}, \mathcal{C}_{1}$ be $(p, q)$-torus curves, $\mathcal{C}_{1}$ being generic. Then, there is a continuous path $\gamma:[0,1] \rightarrow \mathbb{P}_{p q}\left(\mathbb{P}_{d}\right.$ is the projective space of all curves of degree $d$ ) such that if we denote $\mathcal{C}_{t}:=\gamma(t)$, then $\mathcal{C}_{t}$ is a generic $(p, q)$-torus curve, $\forall t \in(0,1]$.

We apply the following result which can be found in Dimca [3] or Zariski [9].

Proposition 2.3 Let $\gamma:[0,1] \rightarrow \mathbb{P}_{d}$ be a continuous map and denote $\mathcal{C}_{t}:=\gamma(t)$. Assume that $\mathcal{C}_{t}$ are equisingular for all $t \in(0,1]$.

(D1) If $\mathcal{C}_{0}$ is also equisingular, then $\mathcal{C}_{0}$ and $\mathcal{C}_{1}$ are isotopic and hence $\pi_{1}\left(\mathbb{P}^{2} \backslash \mathcal{C}_{0}\right) \cong$ $\pi_{1}\left(\mathbb{P}^{2} \backslash \mathcal{C}_{1}\right)$.

(D2) If $\mathcal{C}_{0}$ is reduced, then there is a natural epimorphism

$$
\pi_{1}\left(\mathbb{P}^{2} \backslash \mathcal{C}_{0}\right) \stackrel{\rho\left(\mathcal{C}_{1}, \mathcal{C}_{0}\right)}{\longrightarrow} \pi_{1}\left(\mathbb{P}^{2} \backslash \mathcal{C}_{1}\right)
$$

defined as

$$
\pi_{1}\left(\mathbb{P}^{2} \backslash \mathcal{C}_{0}\right) \stackrel{i_{*}}{\cong} \pi_{1}\left(\mathbb{P}^{2} \backslash R\left(\mathcal{C}_{0}\right)\right) \stackrel{i_{*}}{\longrightarrow} \pi_{1}\left(\mathbb{P}^{2} \backslash \mathcal{C}_{t}\right) \stackrel{(\mathrm{D} 1)}{\cong} \pi_{1}\left(\mathbb{P}^{2} \backslash \mathcal{C}_{1}\right),
$$

where $R(\mathcal{C})$ means a regular neighborhood and the maps denoted by $i$ are inclusions.

Directly from Theorem 1.5 and Propositions 2.2 and 2.3, the following holds. 
Corollary 2.4 There is a natural epimorphism $\mathbb{Z} / p \mathbb{Z} * \mathbb{Z} / q \mathbb{Z} \rightarrow \pi_{1}\left(\mathbb{P}^{2} \backslash \mathcal{C}\right)$ for any generic $(p, q)$-torus curve $\mathcal{C}$.

The final step involves orbifold maps. Let us recall the definitions.

Definition 2.5 An orbifold $X_{\varphi}$ is a quasiprojective Riemann surface $X$ with a function $\varphi: X \rightarrow \mathbb{N}$ taking value 1 outside a finite number of points.

Definition 2.6 For an orbifold $X_{\varphi}$, let $p_{1}, \ldots, p_{n}$ be the points such that $m_{j}:=$ $\varphi\left(p_{j}\right)>1$. Then, the orbifold fundamental group of $X_{\varphi}$ is

$$
\pi_{1}^{\text {orb }}\left(X_{\varphi}\right):=\pi_{1}\left(X \backslash\left\{p_{1}, \ldots, p_{n}\right\}\right) /\left\langle\mu_{j}^{m_{j}}=1\right\rangle,
$$

where $\mu_{j}$ is a meridian of $p_{j}$. We denote $X_{\varphi}$ by $X_{m_{1}, \ldots, m_{n}}$.

Definition 2.7 Let $X_{\varphi}$ be an orbifold and $Y$ a smooth algebraic variety. A dominant algebraic morphism $\rho: Y \rightarrow X$ defines an orbifold morphism $Y \rightarrow X_{\varphi}$ if for all $p \in X$, the divisor $\rho^{*}(p)$ is a $\varphi(p)$-multiple.

The following proposition has been proved by Catanese, Keum and Oguiso [2] and the two first named authors [1].

Proposition 2.8 Let $\rho: Y \rightarrow X$ define an orbifold morphism $Y \rightarrow X_{\varphi}$. Then $\rho$ induces a morphism $\rho_{*}: \pi_{1}(Y) \rightarrow \pi_{1}^{\text {orb }}\left(X_{\varphi}\right)$. Moreover, if the generic fiber is connected, then $\rho_{*}$ is surjective.

Proposition 2.9 Let $\mathcal{C}$ be a $(p, q)$-torus curve $\mathcal{C}$. Then there exists a natural epimorphism $\rho_{\mathcal{C}}: \pi_{1}\left(\mathbb{P}^{2} \backslash \mathcal{C}\right) \rightarrow \mathbb{Z} / p \mathbb{Z} * \mathbb{Z} / q \mathbb{Z}$. Moreover, if $\mathcal{C}_{0}, \mathcal{C}_{1}$ are $(p, q)$-torus curves, $\mathcal{C}_{1}$ generic, then the map $\rho\left(\mathcal{C}_{1}, \mathcal{C}_{0}\right)$ in (2-1) satisfies

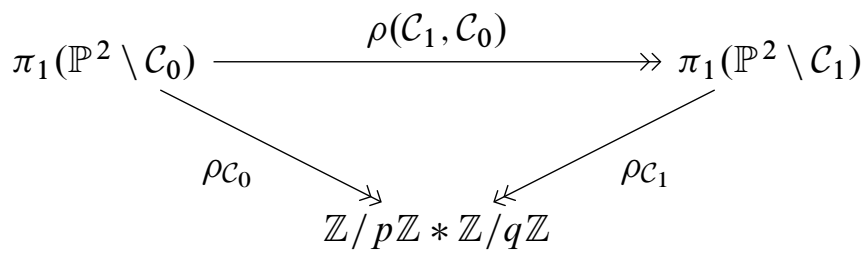

Proof For a curve $\mathcal{C}$ the map $\rho_{\mathcal{C}}$ comes from the orbifold map $\mathbb{P}^{2} \backslash \mathcal{C} \rightarrow \mathbb{P}_{p, q}^{1} \backslash\{[1:-1]\}$ given by $[x: y: z] \mapsto\left[f_{p}^{q}: f_{q}^{p}\right]$. The genericity guarantees that the generic fiber is irreducible. The last statement comes from the fact that the orbifold map can be put in a family.

Remark 2.10 There is a slight ambiguity in Proposition 2.9, since a torus curve may admit several decompositions. The last statement is true if the deformation respects the decompositions. 
Let $\mathcal{C}_{0}$ be a curve with Equation (1-1), for $N=p, a+b=d=q$. Then, by Theorem 1.5, the map $\varphi:=\rho_{\mathcal{C}_{1}} \circ \rho\left(\mathcal{C}_{1}, \mathcal{C}_{0}\right)$ defines an epimorphism

$$
\mathbb{Z} / p \mathbb{Z} * \mathbb{Z} / q \mathbb{Z} \rightarrow \mathbb{Z} / p \mathbb{Z} * \mathbb{Z} / q \mathbb{Z}
$$

Theorem 2.11 The fundamental group of any generic $(p, q)$-curve is isomorphic to the free product $\mathbb{Z} / p \mathbb{Z} * \mathbb{Z} / q \mathbb{Z}$.

Proof It is enough to prove that (2-3) is an isomorphism which is a consequence of the fact that $\mathbb{Z} / p \mathbb{Z} * \mathbb{Z} / q \mathbb{Z}$ is a Hopfian group; see Karrass and Solitar [4].

\section{References}

[1] E Artal Bartolo, J I Cogolludo-Agustín, On the connection between fundamental groups and pencils with multiple fibers, J. Singul. 2 (2010) 1-18 MR2763015

[2] F Catanese, J Keum, K Oguiso, Some remarks on the universal cover of an open K3 surface, Math. Ann. 325 (2003) 279-286 MR1962049

[3] A Dimca, Singularities and topology of hypersurfaces, Universitext, Springer, New York (1992) MR1194180

[4] A Karrass, D Solitar, On free products, Proc. Amer. Math. Soc. 9 (1958) 217-221 MR0095875

[5] V S Kulikov, V M Kharlamov, On braid monodromy factorizations, Izv. Ross. Akad. Nauk Ser. Mat. 67 (2003) 79-118 MR1992194

[6] A Némethi, On the fundamental group of the complement of certain singular plane curves, Math. Proc. Cambridge Philos. Soc. 102 (1987) 453-457 MR906619

[7] M Oka, Some plane curves whose complements have non-abelian fundamental groups, Math. Ann. 218 (1975) 55-65 MR0396556

[8] E R van Kampen, On the fundamental group of an algebraic curve, Amer. J. Math. 55 (1933) 255-267 MR1506962

[9] O Zariski, On the problem of existence of algebraic functions of two variables possessing a given branch curve, Amer. J. Math. 51 (1929) 305-328 MR1506719

EAB, JICA: Departamento de Matemáticas, IUMA, Universidad de Zaragoza

C/ Pedro Cerbuna 12, 50009 Zaragoza, Spain

JO-G: Centro Universitario de la Defensa-IUMA, Universidad de Zaragoza Academia General Militar, Ctra de Huesca s/n, 50090 Zaragoza, Spain artal@unizar.es, jicogo@unizar.es, jortigas@unizar.es http://riemann. unizar.es/geotop/WebGeoTo/Profes/eartal/, http://riemann.unizar.es/ jicogo/, http://riemann.unizar.es/ jortigas/

Received: 16 January 2012 Revised: 23 March 2012 